\title{
Einleitung, Patientengut und Methode
}

\section{$1.1 \quad$ Einleitung}

\subsubsection{Hinführung zum Thema}

Die akut aufgetretene, einseitige, idiopathische sensorineurale Hörminderung (,idiopathic sudden sensineural hearing loss“, ISSNHL) ist eine Erkrankung, die das Befinden der betroffenen Erkrankten in der Regel erheblich einschränkt und sie meist auch recht schnell dazu veranlasst, ärztliche Hilfe aufzusuchen. Meistens wird derzeit weltweit, zumindest als Erstmaßnahme, eine systemische Kortikoidtherapie durchgeführt, sei es in oraler, in der Regel jedoch in parenteraler (intravenöser) Form [5, $11,14,16,18,26,30,32,33,36,40,46,57,63,65,71-73,77,78,85,92,93,97$, 106-108, 111].

Die explorative Tympanoskopie inkl. Abdeckung des runden/ovalen Fensters mit autologem Bindegewebe stellt dabei eine andere, für diese Krankheit mögliche Therapieform dar, die bis heute insbesondere dann in Betracht gezogen wird, wenn der Verdacht auf eine der akuten Hörminderung zugrunde liegende Perilymphfistel (PLF) besteht oder aber mit einer initial systemischen Kortikoidapplikation keine ausreichende Hörverbesserung erreicht werden konnte. Dann könnte der operative Mittelohreingriff - so wie er in gleichartigen Fällen für die intratympanalen Kortikoidinjektionen als sog. Salvage Therapy bezeichnet wird - in gleicher Weise als Salvage Surgery eingestuft werden.

\subsubsection{Definition Hörsturz}

Um einen plötzlich aufgetretenen, einseitigen Hörverlust sachgerecht als Hörsturz klassifizieren zu können, muss, dem US National Institute for Deafness and Communication Disorders folgend, neben der Höreinschränkung als 
weiteres Kriterium erfüllt sein, dass sich das Hörvermögen einseitig im Tonschwellenaudiogramm in drei aufeinander folgenden Frequenzen um mehr als $30 \mathrm{~dB}$ innerhalb von 3 Tagen entwickelt hat [1]. Zusätzlich gilt, dass die Höreinbuße rein sensorineuralen Ursprungs $\mathrm{zu}$ sein hat und keine Ursache für den eingetretenen Hörverlust evident sein darf. Ein begleitender Tinnitus und auch Schwindel dürfen zeitgleich mit dem Hörverlust aufgetreten sein sein [3, 9, $10,12,19,22,28,29,30,31,33,38,43,57,61,65,75,78,88,89,97,101]$.

\subsubsection{Hörsturz - Perilymphfistel}

Blair und Simmons waren die ersten, die als eine der zahlreichen Ätiologien für das Auftreten einer akuten unilateralen, innenohrbedingten Hörstörung auch auf das Vorliegen einer Perilymphfistel aufmerksam gemacht haben [6]. Bei dieser Erkrankung kommt es zu einem Leck zwischen den Innenräumen der Kochlea und dem Tympanon, meist im Bereich des runden Fensters mit Abfließen von Perilymphe in das Mittelohr (Tab. 1.1). Dieses Krankheitsbild ist, folgt man den Literaturangaben, typischerweise durch eine besonders ausgeprägte Schwerhörigkeit bis hin zur Taubheit sowie einen in der Regel gleichzeitig, heftig einsetzenden Schwindel und einen begleitenden Tinnitus gekennzeichnet. Aktuelle Lebenssituationen, die mit einer akuten Erhöhung des intrazerebralen oder abrupten Veränderungen des atmosphärischen Druckes einhergehen, wie es bei heftigen Nies- und Hustenattacken, schwerem Heben mit Betätigen der Bauchpresse, Barotraumen und Schädel-Hirn-Traumen vorkommt, sollen als Ursachen für das Einreißen der Rundfenstermembran verantwortlich gemacht werden können [3, 6, 9, 12, 15, 22, 23, 24, 28-30, 33, 37, 38, 43, 57, 59, 61, 65, 71, 75, 78, 84, 87-89, 97, 99, 101]. Als Therapie der Wahl ist bei diesen Krankheitsfällen eine explorative Tympanoskopie mit Abdeckung des runden, ggf. auch ovalen Fensters mit autologem Bindegewebe indiziert, verspricht dieser chirurgische Eingriff doch, den vorhandenen Defekt kausal anzugehen, indem das Leck zwischen dem Innen- und Mittelohr abgedichtet wird.

\subsubsection{Historische Entwicklung der Indikation zur explorativen Tympanoskopie}

Es verwundert nicht, dass bislang mangels ausreichend sicherer, auf nichtinvasivem Wege einsetzbarer Diagnosemöglichkeiten zur Aufdeckung einer PLF von den Klinikern bei Patienten mit hochgradigen „Hörstürzen“ an die Möglichkeit des Vorliegens eines derartigen Lecks zwischen dem Mittel- und Innenohrraum gedacht wurde und diese Erkrankten dann auch nach einer mehr oder weniger langen, erfolglosen systemischen Kortikoidtherapie, wie sie routinemäßig beim Hörsturz eingesetzt wird, schließlich einer explorativen Tympanoskopie unterzogen wurden. Goodhill empfahl 1973 als Erster, dass bei Patienten mit einer Anamnese, die auf eine PLF hindeutet, und beim Ausbleiben einer Spontan- 
Tab. 1.1 Pathophysiologische Entstehungsmechanismen einer Perilymphfistel am runden und ovalen Fenster nach Goodhill 1973 [26]; Explosiver, über einen gestegerten Intrazerebraldruck induzierter Mechanismus (oben) und implosiver, über eine plötzliche Atmosphärendrucksteigerung induzierter Mechanismus (unten)

\section{Entstehungsmechanismen einer Perilymphfistel nach Goodhill}

\section{Explosionsmechanismus}

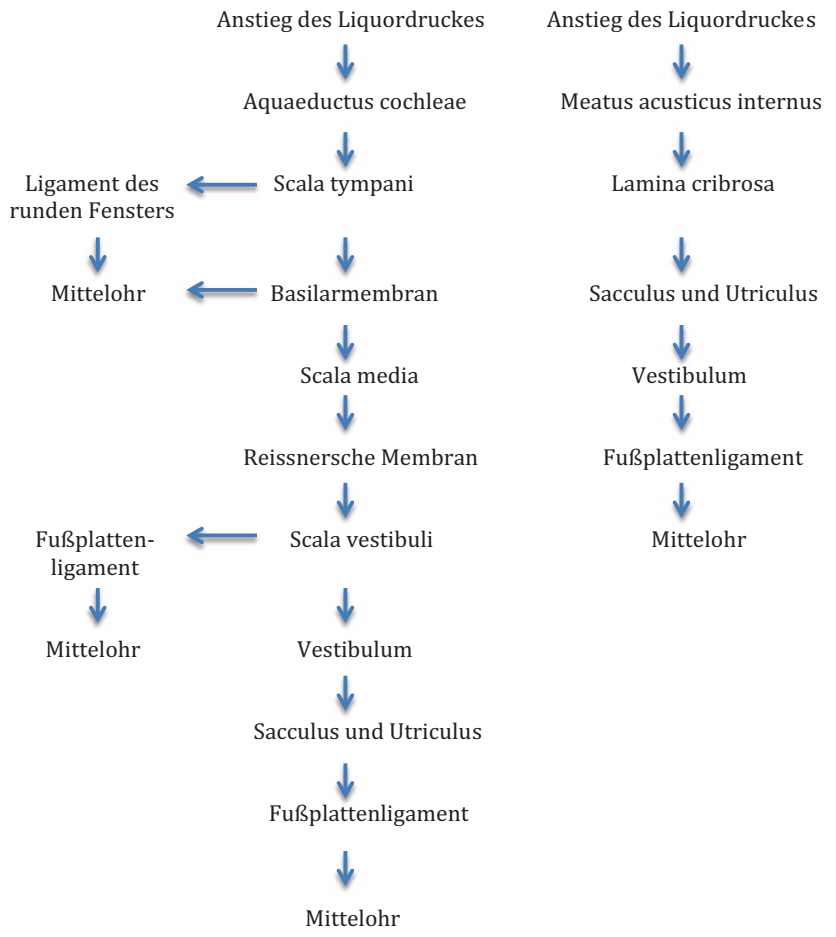

Implosionsmechanismus

Anstieg des Atmosphärendruckes

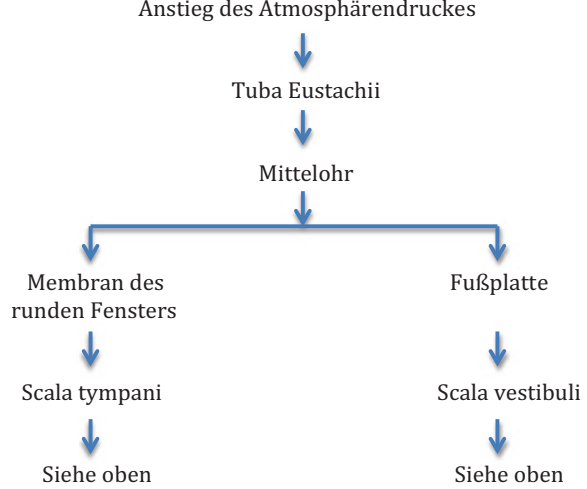


erholung des Hörvermögens unter Bettruhe eine Tympanoskopie durchgeführt werden sollte [23]. Mattox und Simmons erweiterten das Indikationsspektrum 1977 dahin gehend, dass sie auch dann eine Tympanoskopie empfahlen, wenn sich 10 Tage nach einem Hörsturz das Hörvermögen nicht gebessert hatte, auch wenn seitens des Patienten anamnestisch keine Hinweise geäußert worden waren, die auf die Entstehung einer PLF gedeutet hätten [59]. Lamprecht verwies als nächsten Schritt in der Erweiterung des Indikationsspektrums der explorativen Tympanoskopie darauf, dass er eine Indikation $\mathrm{zu}$ diesem chirurgischen Eingriff bereits dann als gerechtfertigt ansah, wenn eine plötzliche Ertaubung mit Schwindel einhergeht, der Hörsturz unter konservativer Therapie keine Besserungstendenz oder sogar im Verlauf eine weitere Verschlechterung zeigt oder aber zeitversetzt noch Schwindel als Symptom hinzutritt [43]. Eine sofortige Operation führte er durch, wenn es anhand der Anamnese und dem Auftreten der klinischen Symptome eindeutige Hinweise für das Entstehen einer Leckage zwischen dem Innen- und Mittelohr gab. Eine Reihe weiterer Autoren haben, insbesondere nach 2010, die Therapie des Hörsturzes mittels Tympanoskopie und Abdeckung des runden/ovalen Fensters mit körpereigenem Bindegewebe zum Thema von Studien gemacht und ihre Ergebnisse publiziert [19, 29-31, 33, 38, 43, $52-54,57,60,61,65,71,75,77,78,81,87,88,97,101]$.

\subsubsection{Fragestellung}

In der vorliegenden Arbeit soll über die Erfahrungen, die wir an der Klinik für HNO-Krankheiten, Kopf- und Halschirurgie am Carl-Thiem-Klinikum in Cottbus im Zeitraum von 2006-2015 mit der explorativen Tympanoskopie und Abdeckung der runden sowie der ovalen Fensternische mit körpereigenem Bindegewebe bei der Behandlung akut aufgetretener sensorineuraler Schwerhörigkeiten gemacht haben, berichtet werden $(n=51)$. Dabei werden einerseits die epidemiologischen Daten, anamnestischen Angaben der Patienten zum Zeitpunkt der Klinikaufnahme, Befunde der ärztlichen Erstuntersuchung zum Ausmaß des initialen Hörverlustes und die postoperativen Behandlungsergebnisse zueinander in Beziehung gesetzt. Unsere Beobachtungen sollen andererseits vergleichend mit den Ergebnissen der mit der gleichen operativen Methode behandelten Patienten $(n=357)$ von vier anderen Autorengruppen betrachtet werden. Die vorliegende Studie geht außerdem der Frage nach, wie sich die Hörentwicklung ausgehend von der stationären Aufnahme bis zu einem Nachuntersuchungstermin nach durchschnittlich 2,2 Jahren in verschiedenen Zeitabschnitten während und nach der Therapie darstellt. Darüber hinaus sollen unsere Behandlungsergebnisse auch Therapieresultaten gegenübergestellt werden, die mit intratympanalen Kortikoidinjektionen, der geläufigsten als Ultima Ratio beim Hörsturz eingesetzten Behandlungsmethode, erreicht wurden $(n=1404)$. 


\subsection{Patientengut und Methode}

\subsubsection{Rekrutierung des Patientengutes}

Für die Auswertung unseres Patientenkollektives haben wir, ausgehend von 76 Patienten, die insgesamt tympanoskopiert wurden, im Sinne einer möglichst homogenen Zusammensetzung unseres Patientenkollektives diejenigen Erkrankten von einer weiteren Auswertung ausgesondert, deren mittlerer anfänglicher Tonhörverlust $<60 \mathrm{~dB}$ betrug (nicht ,profound“) und/oder deren Hörsturzgeschehen mehr als eine Woche vor dem Zeitpunkt der Klinikaufnahme zurücklag $(n=21)$ (Tab. 1.2). Von weiteren 4 Patienten lagen keine ausreichenden audiometrischen Befunde vor, sodass wir auch auf diese Patientengruppe in der weiteren Analyse der Befunde nicht zurückgreifen konnten.

Wir sahen uns dann mit der Situation konfrontiert, dass bei Fehlen offensichtlicher Ätiologien für die Entstehung der akuten schallempfindungsbedingten Hörminderung bis zum Zeitpunkt der Durchführung der Tympanoskopie zunächst die Verdachtsdiagnose eines Hörsturzes gestellt worden war, mit der Durchführung des Mittelohreingriffes aber zutage trat, dass auch einige Patienten pathologische Paukenbefunde aufwiesen, die untereinander aber kein einheitliches pathoanatomisches Schädigungsbild bei dem Mittelohreingriff boten $(n=7)$. Einer dieser Patienten war bereits in einem ersten „Filterungsschritt“" wegen eines bereits knapp 2 Monate zurückliegenden Hörsturzereignisses und einem zugleich anfänglichen Hörverlust von $<60 \mathrm{~dB}$ herausgenommen worden (s. o). Wir haben die Gruppe Erkrankter mit auffälligen Mittelohrbefunden bis auf Weiteres aber in der weiteren statistischen Analyse unseres tympanoskopierten Patientengutes belassen, da ihr Vorkommen ja bei Durchführung einer konservativen Behandlungsmaßnahme gar nicht aufgefallen wäre und sie damit auch weiterhin dem Kollektiv der „Hörsturzpatienten“ zugerechnet worden wären. Es verblieben aus dem Ausgangspool der 76 Patienten damit 51 Patienten, auf die wir zurückgreifen konnten, um personenspezifische Daten, die bei der Aufnahmeuntersuchung erhoben wurden, zum Ausmaß des eingetretenen Hörverlustes in Beziehung zu setzen. Auf weitere 9 Patienten mussten wir bei der Beurteilung der Hörentwicklung nach der Hörsturztherapie verzichten, da von ihnen keine Hördaten mehr im Rahmen einer Nachuntersuchung erhoben werden konnten. Damit verblieben 42 wegen eines Hörsturzes behandelte Erkrankte, die ausreichende Datensätze für die Auswertung der mit der Operation erreichten Hörerfolge boten. Bei 4 von diesen 42 Patienten erbrachte die explorative Tympanoskopie, wie oben beschrieben, dass sie pathologische Befunde im Sinne von vermehrten Flüssigkeitsansammlungen im Bereich der runden Fensternische, Knochenfissuren an der lateralen Kochleawand oder eine Stapesfraktur aufwiesen und deshalb mit einem Perilymphabfluss aus dem Innen- in das Mittelohr gerechnet werden musste. 
Tab. 1.2 Rekrutierung des eigenen Patientengutes

76 wegen eines Hörsturzes tympanoskopierte Patienten

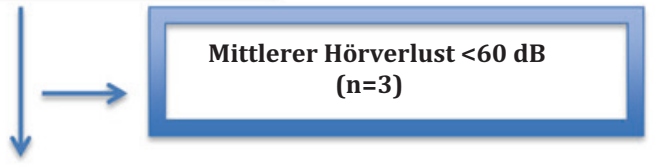

73 wegen eines Hörsturzes

tympanoskopierte Patienten

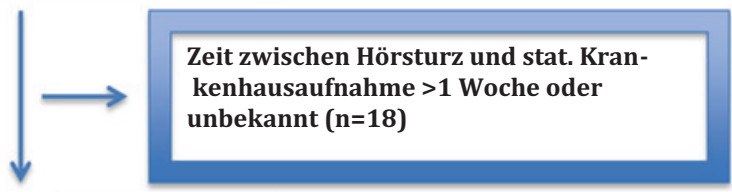

55 wegen eines Hörsturzes

tympanoskopierte Patienten

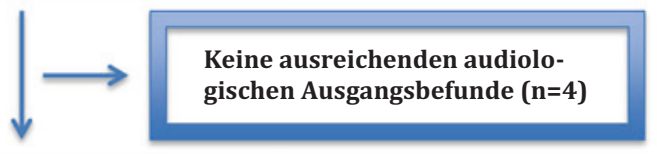

51 wegen eines Hörsturzes

tympanoskopierte Patienten

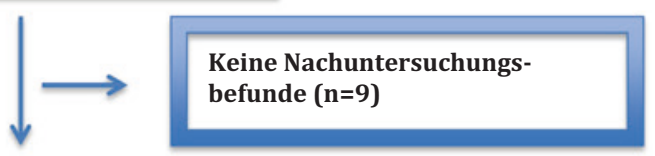

42 wegen eines Hörsturzes

tympanoskopierte Patienten

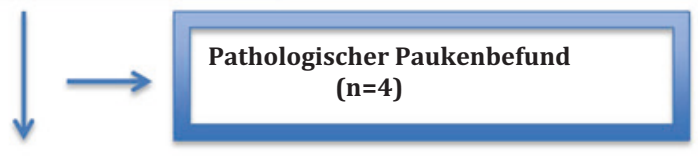

38 wegen eines Hörsturzes

tympanoskopierte Patienten 


\subsubsection{Klinische Vorgehensweise}

Eine erste audiologische Befunderhebung war naturgemäß erst unmittelbar in Zusammenhang mit der stationären Aufnahme der Patienten möglich. Fälschlicherweise (Begründung s. u.) bezeichnen wir das Hörvermögen zum Zeitpunkt des Aufsuchens der Klinik im Weiteren als „Ausgangs- oder Anfangshörverlust“. Der audiologische Befund wurde am Tag der stationären Aufnahme, spätestens jedoch am Folgetag angefertigt. Wir verwendeten die Tonschwellenaudiometrie, bei der über Knochen- und Luftleitung das Hörvermögen in den Frequenzen zwischen 0,25 und $8 \mathrm{kHz}$ gemessen wurde. Im Mittel war ein Zeitintervall von 2,69 Tagen (Variationsbreite 0-10 Tage) zwischen dem Hörsturzereignis und der Krankenhausaufnahme verstrichen. Es folgte dann während einer mehr oder weniger langen Periode eine systemischen Kortikoidtherapie nach dem sog. Stennert-Schema: tägliche Kortisongaben (Predisolut ${ }^{\circledR}$ ) in absteigender Dosierung, d. h., 1. Tag (250 mg), 2. Tag (250 mg), 3. Tag (150 mg), ab 4. Tag (150 mg) bis auf 2,5 mg (Tablettenform) ab 16. Tag, unter täglich mehrfacher Beobachtung der Blutzuckerwerte [62]. Zusätzlich wurde die Gabe von Pentoxifyllin angesetzt, beginnend mit $600 \mathrm{mg} / 500 \mathrm{ml}$ isotoner NaCl-Lösung/d und Dosisreduktion ab dem 4. Tag auf $300 \mathrm{mg}$ Pentoxifyllin/500 $\mathrm{ml}$ isotone $\mathrm{NaCl}$-Lösung/d bis zum 10. Tag und ggf. auch darüber hinaus. Weitere audiologische Kontrolluntersuchungen folgten bis $\mathrm{zu}$ dem individuell bestimmten und nicht an eine Regel gebundenen Termin der Tympanoskopie (Mittelwert: 4,83 Tage zwischen Klinikaufnahme und Operation; Variationsbreite: 1-16 Tage). Es wurden auch postoperativ weitere tonschwellenaudiometrische Untersuchungen, teilweise noch unter stationären Kautelen, bis zum 20. Tag nach dem chirurgischen Eingriff vorgenommen. Eine letzte Hörmessung fand im Rahmen einer Nachuntersuchung bei erneuter Einbestellung der Patienten in die Klinik statt (Durchschnittswert: 2,2 Jahre nach dem Hörsturzereignis; Variationsbreite: 22 Tage bis 12,9 Jahre).

\subsubsection{Technik der Tympanoskopie}

Im Rahmen einer Tympanoskopie, bei der die Eröffnung der Mittelohrräume routinemäßig wie bei einer hörverbessernden Operation transmeatal in Vollnarkose vorgenommen wurde, wurden sowohl die runde als auch die ovale Fensternische inspiziert und unabhängig von dem intraoperativen Befund wurden beide mit autologem Bindegewebe obliteriert. Manipulationen im knöchernen Bereich der Rundfenstermembran (Abfräsen überhängender Knochenstrukturen) wurden nicht vorgenommen, sodass diese in der Regel nicht komplett, sondern nur die dazugehörige Fensternische, eingesehen werden konnte. 


\subsubsection{Untergruppenbildung}

Im Rahmen der Auswertung unseres Patientengutes wurde das Gesamtpatientenkollektiv verschiedentlich in (meist zwei) Subpopulationen unterteilt, bei denen die Patienten jeweils einer der Untergruppen entsprechend zugeordnet wurden, je nachdem, welches der vorgegebenen Filterkriterien sie erfüllten oder aber nicht erfüllten.

\subsubsection{Berechnung der Hörverluste}

Neben der Auswertung der frequenzbezogenen Hörminderungen wurde auch ein mittlerer Hörverlust berechnet, der dem arithmetischen Mittelwert der Hörminderungen aus den Frequenzen $500 \mathrm{~Hz}, 1 \mathrm{kHz}, 2 \mathrm{kHz}$ und $4 \mathrm{kHz}\left(\mathrm{PTA}_{4}\right)$ entspricht. In Einzelfällen, auf die an entsprechender Stelle besonders hingewiesen wird, wurden der Auswertung auch andere Algorithmen in der Beurteilung des Hörverlustes zugrunde gelegt. Bei einer absoluten Taubheit mit keinerlei messbaren Hörreaktionen im Tonschwellenaudiogramm wurde metrisch der Hörverlust mit $120 \mathrm{~dB}$ angegeben.

\subsubsection{Hörgewinn nach Kanzaki}

Ein Vorschlag zur Beschreibung der Hörgewinne basiert auf Kanzaki, der für die Einteilung der Hörverbesserungen nach einer Therapie eine Kategorienbildung vorschlug, die sich auf das Ausmaß des Hörgewinns bezieht [35]. Hörgewinne, bei denen das Hörvermögen der gesunden Gegenseite wieder erreicht wurden, wurden als komplett (,complete“) gekennzeichnet, solche von $>30 \mathrm{~dB}$ als deutlich ausgeprägt (,marked“) definiert. Hörgewinne zwischen $10 \mathrm{~dB}$ und $30 \mathrm{~dB}$ wurden als geringgradig (,slight“) bezeichnet und solche von $<10 \mathrm{~dB}$ oder Fälle, bei denen sich sogar weitere Hörverluste in der Folgezeit nach dem stationären Krankenhausaufenthalt eingestellt hatten, wurden als fehlende (,no change“) oder gar negative Behandlungserfolge beschrieben.

\subsubsection{Bestimmung der Gleichgewichtsfunktion}

Bei der Bestimmung der Gleichgewichtsfunktion wurde eine manuelle Auswertung der elektronystagmografisch ermittelten Nystagmusbefunde vorgenommen. Es wurden die Frequenzwerte/30 s vom Spontannystagmus (SPN), einer Kalorisation mit $30^{\circ} \mathrm{C}$ und $44{ }^{\circ} \mathrm{C}$ warmem Wasser und einer Langdrehmethode mit einer trapezförmigen Beschleunigung von $3^{\circ} \mathrm{C} / \mathrm{s}^{2}$ bis $\mathrm{zu}$ einer 
Winkelgeschwindigkeit von $90 \%$ (Perrotatorius) sowie nach 2 min konstanter Drehung einem Stopp mit einer Bremsverzögerung von $360^{\circ} / \mathrm{s}^{2}$ (Postrotatorius) vorgenommen. Aus den Daten der Kalorisation wurde das prozentuale Seitenüberwiegen und aus den beiden Drehphasen das prozentuale Richtungsüberwiegen (,directional preponderance“) der Nystagmusantworten zur weiteren Auswertung gebildet.

\subsubsection{Statistische Auswertung}

Für den Vergleich von zwei Subpopulationen unseres Gesamtpatientengutes setzten wir den Students-T-Test ein, bei nominal skalierten Daten erfolgte die Auswertung anhand von Vier-Felder-Tafeln (Chi-Quadrat-Test) mit Bestimmung der durch sie ermittelten 5-\%- und 0,001-\%-Irrtumswahrscheinlichkeiten. Wurden mehrere unabhängige Variablen (Parameter) aus der Grundgesamtheit hinsichtlich ihrer Zusammensetzung gegenüber einer metrisch skalierten, abhängigen Variable (Hörwerte in Dezibel) parallel untersucht, kam die Varianzanalyse (ANOVA) zur Anwendung. Lagen sowohl die unabhängigen als auch die abhängigen Parameter nominal skaliert vor, wurde für den Vergleich der Kruskal-Wallis-Test verwendet.

Bei allen Testergebnissen, deren Resultate hochsignifikante Wahrscheinlichkeitsmaße in den Unterschieden der Subpopulationen aus mehreren Gruppenvergleichen beinhalteten, wurden in einem zweiten Auswertungsschritt eine Posthoc-Bonferroni-Adjustierung, die als konservativ eingeschätzt wird, und auch eine Korrektur nach Li vorgenommen [21, 48, 55]. Besonders das zweite der beiden aufgeführten Verfahren soll sich dabei durch eine sehr große „Power“ auszeichnen [55].

Für die Überprüfung von Zusammenhängen zwischen zwei Parametern kam die Bildung des Korrelationskoeffizienten (bei metrisch und nominal skalierte Daten) zur Anwendung [21].

Die Ergebnisse verschiedener vergleichender Analysen wurden bezüglich der Güte ihrer Aussagekraft (Irrtumswahrscheinlichkeit) auf ihre 5-\%- und 0,1-\%-Signifikanzschwelle (signifikant und hochsignifikant) hin überprüft.

\subsubsection{Gewichteter Mittelwert}

Bei dem Vergleich unserer Resultate mit denen verschiedener anderer Studiengruppen, die aus einer Literaturrecherche zusammengetragen wurden, wurde bei der Zusammenfassung der Messdaten ein gewichteter Mittelwert gebildet, der die Anzahl der Patienten, die eine Studiengruppe zu dem Gesamtergebnis beigesteuert hat, entsprechend berücksichtigt. 


\subsubsection{Abkürzungen}

Die Abkürzung „SAW“ wird im Folgenden für die Standardabweichung und die Abkürzung „n“ für die Anzahl der in eine statistische Auswertung einbezogenen Patienten gewählt.

\section{$1.3 \quad$ Zusammenfassung}

Im vorliegenden Kapitel wird zunächst auf den Zusammenhang zwischen einer akut eingetretenen, idiopathischen sensorineuralen Hörminderung (= Hörsturz, ISSNHL) und einer Perilymphfistel (PLF) mit Leckage, die zwischen dem Innenund Mittelohr auftreten kann, eingegangen und in einem historischen Abriss erläutert, dass die bei letztgenanntem Krankheitsbild kausal angreifende Therapie einer explorativen Tympanoskopie mit Abdichtung der runden/ovalen Fenstermembran in den vergangenen Jahrzehnten schrittweise auch unter bestimmten Bedingungen als Therapiemethode beim hochgradigen Hörsturz/Taubheit zur Anwendung kam.

Die Methode der systemischen Kortikoidbehandlung bei den von uns behandelten Hörsturzerkrankten und auch das Verfahren der explorativen Tympanoskopie werden beschrieben.

Anhand eines Kollektives aus 51 in unserer Klinik operierten Patienten mit ISSNHL und der Sammlung von Daten aus vier weiteren, aus der Literatur recherchierten Studien (Gesamtzahl $=357$ Patienten), bei denen ein gleichartiges therapeutisches Vorgehen gewählt worden war, wird einerseits untersucht, welche epidemiologischen Parameter, anamnestischen Angaben und klinischen Anfangsbefunde die Höhe des Ausgangshörverlustes beeinflusst haben können. Andererseits wird bei 42 durch uns behandelten Hörsturzerkrankten die postoperative Hörentwicklung, entweder gemittelt aus Hörwerten des Tonschwellenaudiogramms bei $0,5 \mathrm{kHz}, 1 \mathrm{kHz}, 2 \mathrm{kHz}$ und $4 \mathrm{kHz}\left(\mathrm{PTA}_{4}\right)$ oder anhand der sog. Kanzaki-Kriterien, bestimmt und $\mathrm{zu}$ den individualspezifisch erhobenen Patientendaten in Beziehung gesetzt, mit dem Ziel, Hinweise dafür zu erhalten, welche Parameter für den Eintritt eines behandlungsbedingten Hörerfolges Prognoserelevanz besitzen. Auch diese Beobachtungen werden mit den Ergebnissen aus anderen Publikationen verglichen.

Die Hörergebnisse, die mit der explorativen Tympanoskopie inkl. Abdichtung der runden/ovalen Fensternischen erzielt werden konnten, werden schließlich Studienresultaten gegenübergestellt, die laut Literaturmitteilungen mit intratympanalen Kortikoidinjektionen, einem bei der Hörsturzbehandlung weithin verbreiteten und als alternativ zur explorativen Tympanoskopie anzusehenden Therapieverfahren, erreicht worden waren $(n=1404)$.

Als statistische Verfahren kommen bei den einzelnen Auswertungsschritten wiederholt der Student's-T-Test, der Chi-Quadrat-Test und Korrelationsuntersuchungen zur Anwendung, mithilfe derer Signfikanzbestimmungen auf dem 5-\%- 
und 0,1-\%-Niveau durchgeführt wurden. Die Testergebnisse hatten zusätzlich den Kriterien einer Post-hoc-Bonferroni- und auch einer Li-Adjustierung standzuhalten. Bei gleichzeitigem Vergleich mehrerer Untersuchungsgruppen mit nominal skalierten, unabhängigen Variablen wurden die arithmetischen Mittelwerte der beiden abhängigen Variablen gebildet und dann die Unterschiede der Populationen mittels ANOVA (metrisch skalierte Daten) oder, bei nominal skalierten Daten auch der abhängigen Variablen, der Kruskal-Wallis-Test eingesetzt. Für den Vergleich der Hörergebnisse, die mit Behandlungsverfahren in einer Mehrzahl von Studiengruppen erzielt wurden, wurden gewichtete Mittelwertbildungen vorgenommen.

Open Access Dieses Kapitel wird unter der Creative Commons Namensnennung 4.0 International Lizenz (http://creativecommons.org/licenses/by/4.0/deed.de) veröffentlicht, welche die Nutzung, Vervielfältigung, Bearbeitung, Verbreitung und Wiedergabe in jeglichem Medium und Format erlaubt, sofern Sie den/die ursprünglichen Autor(en) und die Quelle ordnungsgemäß nennen, einen Link zur Creative Commons Lizenz beifügen und angeben, ob Änderungen vorgenommen wurden.

Die in diesem Kapitel enthaltenen Bilder und sonstiges Drittmaterial unterliegen ebenfalls der genannten Creative Commons Lizenz, sofern sich aus der Abbildungslegende nichts anderes ergibt. Sofern das betreffende Material nicht unter der genannten Creative Commons Lizenz steht und die betreffende Handlung nicht nach gesetzlichen Vorschriften erlaubt ist, ist für die oben aufgeführten Weiterverwendungen des Materials die Einwilligung des jeweiligen Rechteinhabers einzuholen.

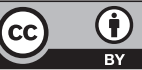

